\title{
Armodafinil-induced wakefulness in animals with ventrolateral preoptic lesions
}

This article was published in the following Dove Press journal:

Nature and Science of Sleep

2 May 2014

Number of times this article has been viewed

\section{Ramalingam Vetrivelan Clifford B Saper \\ Patrick M Fuller}

Department of Neurology, Harvard Medical School and Beth Israel Deaconess Medical Center, Boston, MA, USA
Correspondence: Patrick M Fuller Department of Neurology, Beth Israel Deaconess Medical Center, 3 Blackfan Avenue, E/CLS 707, Boston, MA 02215, USA

Tel + I 6177352811

Fax + I 6177352910

Email pfuller@bidmc.harvard.edu

\begin{abstract}
Armodafinil is the pharmacologically active R-enantiomer of modafinil, a widely prescribed wake-promoting agent used to treat several sleep-related disorders including excessive daytime sleepiness associated with narcolepsy, shift work sleep disorder, and obstructive sleep apnea/hypopnea syndrome. Remarkably, however, the neuronal circuitry through which modafinil exerts its wake-promoting effects remains unresolved. In the present study, we sought to determine if the wake-promoting effects of armodafinil are mediated, at least in part, by inhibiting the sleep-promoting neurons of the ventrolateral preoptic (VLPO) nucleus. To do so, we measured changes in waking following intraperitoneal administration of armodafinil $(200 \mathrm{mg} / \mathrm{kg})$ or the psychostimulant methamphetamine $(1 \mathrm{mg} / \mathrm{kg})$ in rats with cell-body specific lesion of the VLPO. Rats with histologically confirmed lesions of the VLPO demonstrated a sustained increase in wakefulness at baseline, but the increase in wakefulness following administration of both armodafinil and methamphetamine was similar to that of intact animals. These data suggest that armodafinil increases wakefulness by mechanisms that extend beyond inhibition of VLPO neurons.
\end{abstract}

Keywords: EEG, sleep, orexin-saporin, methamphetamine

\section{Introduction}

Modafinil is a wake-promoting agent that is currently approved by the US Food and Drug Administration (FDA) for the treatment of excessive daytime sleepiness associated with narcolepsy, shift work sleep disorder, and obstructive sleep apnea/ hypopnea syndrome. ${ }^{1,2}$ As the wake-promoting effects are due mainly to the R-isomer, armodafinil, this agent has now been introduced for clinical use. ${ }^{3}$ At present, however, little is known about armodafinil's cellular mechanism of action for enhancing arousal. ${ }^{4}$ Nevertheless, several studies have suggested an important role for brain dopaminergic and noradrenergic signaling mechanisms in mediating the wake-promoting effects of the racemic mixture of modafinil in vivo. ${ }^{5-7}$ More recent work has suggested the interesting possibility of a dopamine-dependent adrenergic signaling mechanism of action for modafinil, although this remains largely untested. ${ }^{8}$ Similar to its unresolved mode of action, the central nervous system (CNS) site(s) at which modafinil exerts its wake-promoting effects also remains uncertain. Given its potent wake-promoting properties it would be reasonable to postulate that armodafinil suppresses or facilitates, respectively, the activity of sleep- or wake-promoting systems.

We hypothesize that inhibition of the ventrolateral preoptic nucleus (VLPO) may be a mechanism for mediating the arousal-promoting effects of armodafinil. Neurons of the VLPO are sleep-active and cell-body specific lesions of the VLPO result in profound 
insomnia in rats. ${ }^{9-11}$ During the waking period, sleep-active VLPO neurons are inhibited by inputs from brainstem monoaminergic arousal systems, including both dopaminergic and noradrenergic systems. ${ }^{12}$ Several converging lines of evidence suggest a possible role for the VLPO in mediating the wakepromoting effects of armodafinil: 1) noradrenergic inhibition of the VLPO is potentiated by racemic mixed modafinil in vitro; ${ }^{13} 2$ ) dopamine inhibits the VLPO through activation of $\alpha 2$ adrenoreceptors; ${ }^{14}$ and 3) c-Fos labeling in the VLPO is reduced following modafinil treatment. ${ }^{15}$ Thus, there are ample data to suggest that the VLPO may be a neuroanatomic substrate through which armodafinil mediates its effects on wakefulness. We tested this hypothesis in the present study by evaluating the wake-promoting properties of armodafinil in rats with VLPO lesions.

\section{Methods}

\section{Animals}

Pathogen free adult male Sprague Dawley rats (300-350 g) were housed in individual cages. The cages were set within inside sound-attenuating isolation chambers, which provided ventilation, lighting (12:12 light-dark cycle; light on at $7 \mathrm{am}$ ) and visual isolation. Care of the rats in the experiment met National Institutes of Health standards, as set forth in the Guide for the Care and Use of Laboratory Animals, and all protocols were approved by the Harvard Medical School and Beth Israel Deaconess Medical Center Institutional Animal Care and Use Committees.

\section{Surgery}

Under chloral hydrate anesthesia (7\% in saline, $350 \mathrm{mg} / \mathrm{kg})$, a burr hole was made in the skull and a fine glass pipette (1 mm glass stock, tapering slowly to a $10-20 \mathrm{~mm}$ tip) containing $0.1 \%$ orexin-saporin (OX-SAP) was lowered to the VLPO (coordinates AP -0.6 from bregma, ML \pm 1.00 , DV -8.5 as per the atlas of Paxinos and Watson ${ }^{16}$ ) and 200 $n L$ OX-SAP was injected (VLPOx rats; $n=11$ ). Another set of rats $(n=6)$ received an equivalent volume injection of saline into the VLPO and served as sham-lesioned controls (sham-L). For the collection of sleep-wake data (ie, electroencephalography/electromyography [EEG/EMG] recordings) from these rats, four EEG screw electrodes were implanted into the skull, two each laterally in the frontal and parietal bones, and two flexible EMG wire electrodes were placed into the neck muscles. The free ends of the leads were soldered into a socket that was attached to the skull with dental cement, and the incision was then closed with wound clips. ${ }^{17}$

\section{Sleep-wake recordings and analysis}

After 2 weeks of postoperative recovery, the rats were connected via flexible recording cables to a commutator, which, in turn, was connected to a Grass polygraph and computer. The rats were habituated to the cables for 1 day. Continuous recording of EEG/EMG began after this habituation period and continued for 48 hours or 72 hours, interrupted only by injections (armodafinil, methamphetamine, or vehicle). The digitized EEG/EMG data of each rat were divided into 12 second epochs and visually scored as wake, non-rapid eye movement (NREM) sleep, or rapid eye movement (REM) sleep using established criteria. ${ }^{11}$ Scoring was done before histological examination; scorers were unaware of both the extent of the lesions and the administered drug/vehicle.

\section{Drug administration}

In this study we used armodafinil, which is the isolated $\mathrm{R}$-isomer of modafinil and methamphetamine. Armodafinil was suspended in a vehicle solution (Tween; Cephalon Inc, Frazer, PA, USA) and administered at a dose of $200 \mathrm{mg} / \mathrm{kg}$ bodyweight (bw). Similarly, methamphetamine was dissolved in saline and administered at a dose of $1 \mathrm{mg} / \mathrm{kg}$ bw. All injections were administered at 10 am (early-subjective day) since this corresponds to the time of maximal sleep tendency in the rat's normal rest/sleep period.

\section{Histology}

Animals were sacrificed using deep anesthesia $(500 \mathrm{mg} /$ $\mathrm{kg}$ chloral hydrate) followed by transcardial perfusion with $50 \mathrm{~mL}$ saline, followed by $250 \mathrm{~mL}$ of neutral phosphate buffered formalin (Fischer Scientific Co, Waltham, MA, USA). Brains were removed, incubated in $20 \%$ sucrose at $4^{\circ} \mathrm{C}$, and then sectioned at $40 \mu \mathrm{m}$ on a freezing microtome. To assess the completeness of lesions generated using orexin-saporin, we performed Nissl stains on one series from each brain. This was done by mounting the series on gelatin-coated slides, and washing in $\mathrm{H}_{2} \mathrm{O}$ and then phosphate buffered saline (PBS). Sections were then incubated in $0.25 \%$ thionin in $0.1 \mathrm{M}$ acetate buffer solution for 2 minutes, differentiated in graded ethanols, and delipidated in xylene before being coverslipped. Lesion sites were then identified based on neuronal loss and gliosis, and the lesion size was then quantified by counting the number of remaining neurons in the VLPO. The construction of counting boxes and the counting method were similar to those used in previous studies. ${ }^{10,11}$ Cell profile counts were made bilaterally on three sections (separated by $160 \mu \mathrm{m}$ ) for the VLPO cluster, medial extended VLPO, and dorsal extended VLPO region, by counting only neurons with 
clear nuclei. The profile counts were corrected for overcounting by using the Abercrombie factor. Percentage of cell loss in each of these VLPO subregions was estimated using the following equation:

$$
100-(\mathrm{RN} \times 100 / \mathrm{MCC})
$$

where $\mathrm{RN}$ represents the remaining number of neurons in that subregion of the VLPO in each VLPOx rat and $\mathrm{MCC}$ represents the mean cell count in the same region in sham-L rats.

Only animals with $>70 \%$ cell loss bilaterally in the VLPO cluster were included in the final analysis as VLPO lesions of this magnitude have been shown to produce a profound increase in wakefulness..$^{10,11}$

For c-Fos immunohistochemistry, brain sections were first washed in PBS and incubated in primary antiserum diluted in PBS containing $0.3 \%$ Triton X-100 and $0.2 \%$ sodium azide for 1 day at room temperature. Sections were then washed in PBS and incubated in biotinylated secondary antiserum (against appropriate species IgG, 1:1,000, Vector Laboratories, Burlingame, CA, USA) in PBS for 1-hour, washed in PBS and incubated in $\mathrm{ABC}$ reagents for 1-hour. Sections were then washed again and incubated in a solution of 0.06\% 3,3-diaminobenzidine tetrahydrochloride (DAB; Sigma-Aldrich, St Louis, MI, USA), $0.02 \% \mathrm{H}_{2} \mathrm{O}_{2}$ and $0.05 \%$ cobalt chloride and $0.01 \%$ nickel ammonium sulfate. The c-Fos antibody (1:50,000, AB5, 55kD; EMD Millipore, Billerica, MA, USA) used was a rabbit polyclonal antibody raised against residues 4-17 from human c-Fos, and it stained characteristic patterns of cells in the hypothalamus and cerebral cortex in both waking and sleeping intact rats. ${ }^{15}$

\section{Data analysis}

In order to assess the changes in sleep-wake following VLPO lesions, the percentage of time spent in wake, NREM sleep, and REM sleep during a 24-hour period was calculated for VLPOx rats and compared with those of sham-L animals using an unpaired $t$-test. To determine changes in sleep-wake following administration of the drug/vehicle, we analyzed the first 6 hours (divided into 1 hour bins) of post-injection data from the sham-L and VLPOx rats. The percentage of time spent in wake, NREM, and REM from the six 1 hour bins following armodafinil or methamphetamine administration in the VLPOx rats was compared with the respective bins following vehicle injections in the VLPOx rats using paired $t$-tests and with the respective bins following armodafinil or methamphetamine injections in the sham-L rats using unpaired $t$-tests.

\section{Results}

To test our hypothesis, we compared EEG and behavioral arousal responses to armodafinil (200 mg/kg; the isolated R-isomer of modafinil), methamphetamine $(1 \mathrm{mg} / \mathrm{kg})$ or vehicle injection in both sham-L and VLPOx rats. In preliminary dosing experiments, we found that armodafinil at $200 \mathrm{mg} / \mathrm{kg}$ produced near-equivalent waking (about 3 hours) to that of methamphetamine at $1 \mathrm{mg} / \mathrm{kg}$ and so utilized this dosing scheme for our experiments. At these respective dosages, armodafinil and methamphetamine produced a sustained and highly reproducible increase in waking for about 3 hours after the injections in sham-L rats.

\section{Wake promoting effects of modafinil or methamphetamine in VLPOx rats}

Consistent with our previous reports, rats with histologically verified $>70 \%$ cell loss $(n=11)$ in the VLPO demonstrated significant and sustained increases in baseline waking (approximately 34\%) and a concomitant reduction in both NREM and REM sleep (Figure 1). Following this analysis of baseline sleep-wake recording in VLPOx and Sham-L animals, we evaluated the wake-promoting effects of armodafinil, methamphetamine, and vehicle injections.

VLPOx rats displayed a significant increase in wake for about 3 hours following both armodafinil (paired $t$-test; $t=-3.44, P<0.05 ; t=-8.107, P<0.001$; and $t=-5.229$, $P<0.01$, respectively) and methamphetamine (paired $t$-test; $t=-3.443, P<0.05 ; t=-8.107, P<0.001$; and $t=-3.051, P<0.05$, respectively) administration (Figure $2 \mathrm{~A}$ and $\mathrm{B}$ ) compared with vehicle injections. The percentages of wake during each of the first three 1 hour bins following armodafinil and methamphetamine administration was $100 \%$ in both the intact and in the VLPOx rats. It could be argued that this "ceiling effect" limited our ability to detect differences between the two groups. Wakefulness in the VLPOx rats however also continued to be higher after this 3 hour period compared to sham-L animals, and statistical significance was observed at 5 hours after armodafinil (unpaired $t$-test; $t=2.401, P<0.05$ ) and at 5 and 6 hours after methamphetamine (unpaired $t$-test; $t=4.426, P<0.01$ and $t=-2.358 ; P<0.05$, respectively). In summary, the VLPOx rats showed the same level of wake as the intact rats after armodafinil, but because their baseline level of wakefulness is higher, the percentage increase in wake was smaller. This is consistent with armodafinil in part working by decreasing the firing of VLPO neurons, which of course are already deactivated in VLPOx animals. 

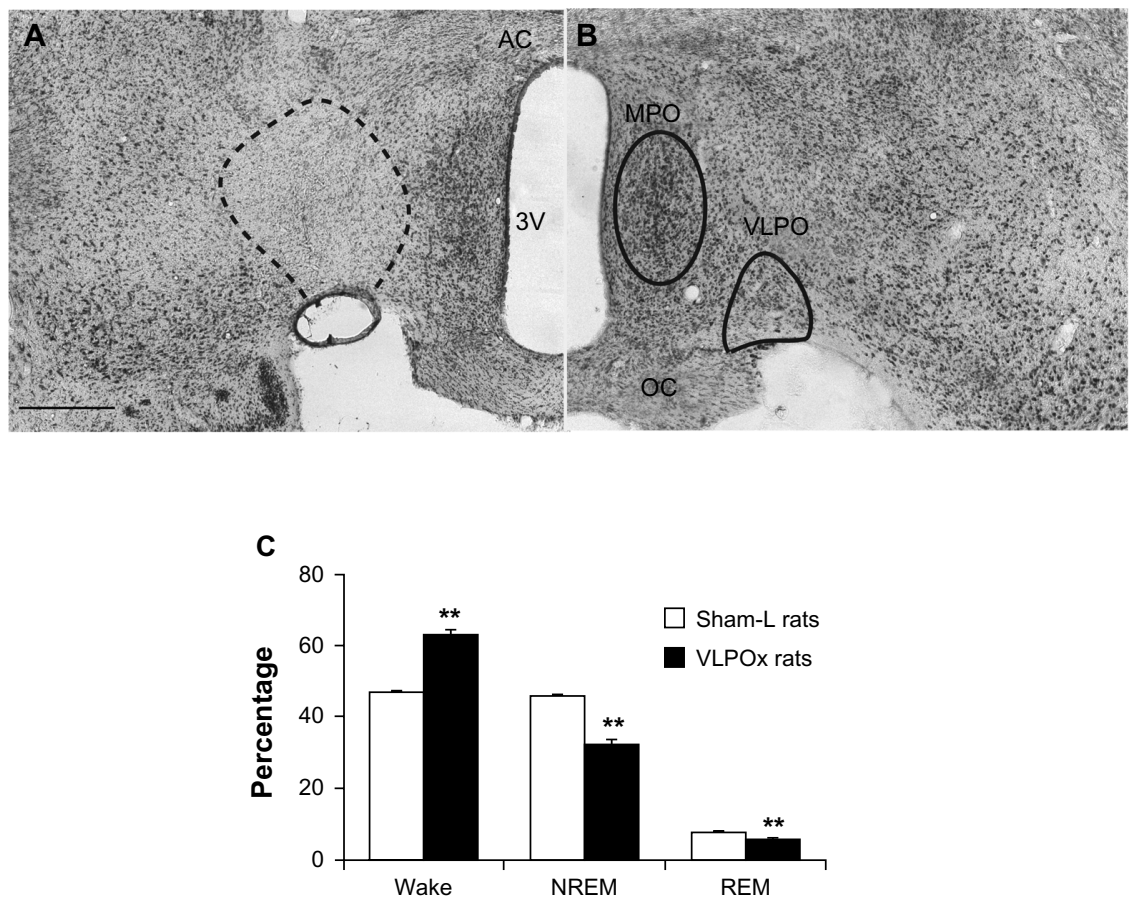

Figure I Histological assessment of VLPOx lesions, which produced a significant increase in wake and concomitant reduction in NREM and REM sleep. Representative photomicrographs of Nissl-stained brain sections from a (A) VLPOx rat and (B) a sham-L rat. (C) Daily percentages of sleep-wake stages (wake, NREM, REM) in sham-L (white bars) and VLPOx rats (black bars). Bilateral lesions of the VLPO produced a significant increase in wake and concomitant decreases in NREM and REM sleep. Notes: Values are mean \pm standard error of the mean; $* * P<0.01$; scale bar: $500 \mu \mathrm{m}$.

Abbreviations: 3V, third ventricle; AC, anterior commissure; MPO, medial preoptic area; NREM, non-REM; OC, optic chiasm; REM, rapid eye movement; sham-L, shamlesioned; VLPO, ventrolateral preoptic area; VLPOx, VLPO-lesioned.

\section{c-Fos response to armodafinil or methamphetamine}

In the present study we also observed marked behavioral differences in the animals following armodafinil versus methamphetamine administration. For example, whereas

A

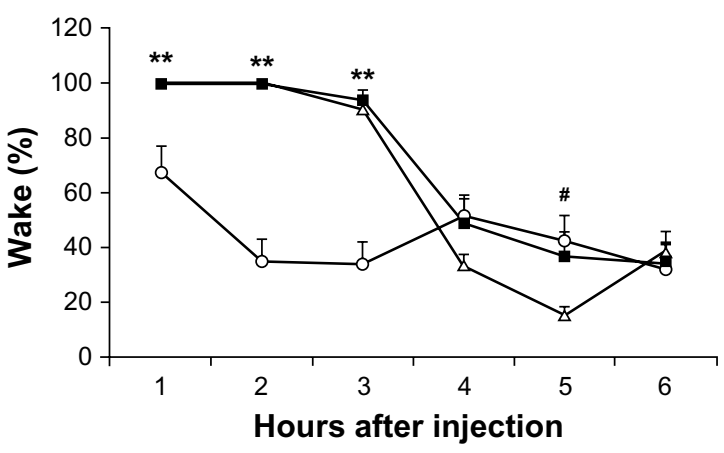

- VLPOx vehicle $\triangle$ Sham-L armodafinil $\rightarrow$ VLPOx armodafinil all of our armodafinil injected rats (VLPOx and Sham-L) showed clear behavioral arousal during the post-injection window the methamphetamine injected rats exhibited more active behaviors, including chewing of bedding and food and general hyperactivity during this same time. c-Fos analysis
B

Methamphetamine

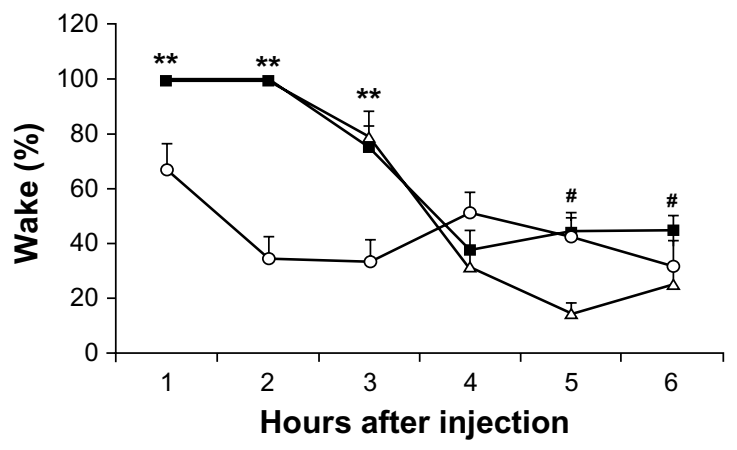

-0 - VLPOx vehicle $\quad \triangle$ Sham-L meth $\rightarrow$ - VLPOx meth

Figure 2 The wake-promoting effects of armodafinil and methamphetamine are not attenuated in VLPOx rats compared to sham-L rats. Both (A) armodafinil and (B) methamphetamine produced a sustained increase in wake for approximately 3 hours in both VLPOx and sham-L rats. A persisting and significant increase in wake was observed into the (A) 5 th hour post-injection hour and the (B) 6 th post-injection hour in VLPOx rats compared to sham-L rats. Wake amounts during the 5th and 6th postinjection hours did not differ between armodafinil and methamphetamine injected VLPOx rats and VLPOx rats receiving vehicle injections.

Notes: Data are mean \pm standard error of the mean; ${ }^{*} * P<0.01$ compared with VLPOx vehicle group; $P<0.01$ when compared with either sham-L armodafinil or sham-L methamphetamine group.

Abbreviations: meth, methamphetamine; sham-L, sham-lesioned; VLPOx, ventrolateral preoptic area-lesioned. 
was therefore performed on the brains of a naive cohort of rats $(\mathrm{n}=12)$ injected with either armodafinil $(200 \mathrm{mg} / \mathrm{kg})$ or methamphetamine $(1 \mathrm{mg} / \mathrm{kg})$. We observed almost no differences across the neuraxis in c-Fos expression between animals receiving armodafinil and those receiving methamphetamine. Comparable c-Fos expression was observed in the cortex (cingulate, insular), thalamic nuclei, basal forebrain, striatum (Figure 3), hypothalamus, amygdala, midbrain (eg, retro-VTA) and brainstem (eg, locus coeruleus) in animals receiving armodafinil versus methamphetamine. Our c-Fos findings in armodafinil injected rats are thus virtually identical to those reported by Scammell et a ${ }^{15}$ in modafinil injected rats.

\section{Discussion}

In light of its potent wake-promoting properties and routine clinical usage in treatment of a spectrum of sleep-wake disorders, it is remarkable that both modafinil's cellular basis
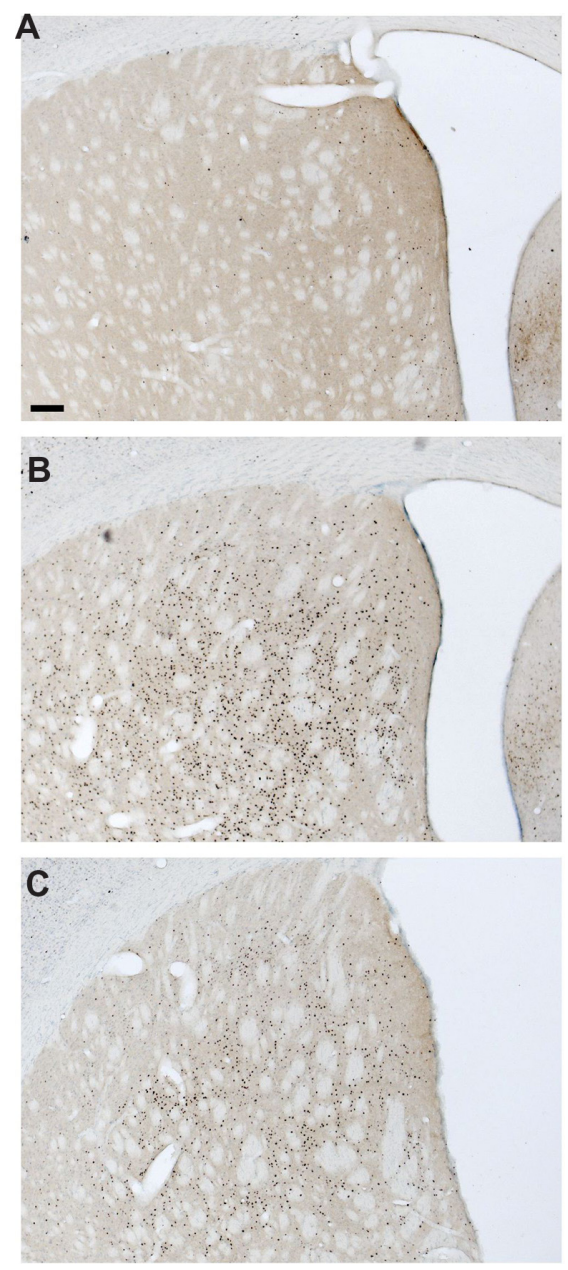

Figure 3 c-Fos expression in the dorsal striatum following armodafinil, methamphetamine, or saline injections.

Notes: Intraperitoneal administration of $(\mathbf{A})$ saline did not induce c-Fos expression in the striatum, whereas intraperitoneal administration of (B) armodafinil $(200 \mathrm{mg} / \mathrm{kg})$ or (C) methamphetamine $(1 \mathrm{mg} / \mathrm{kg})$ induced robust c-Fos expression in the striatum 2 hours post-injection. Scale bar: $200 \mu \mathrm{m}$. of action as well as its neuronal targets remains unresolved. Although most previous studies on the mechanism of modafinil have used the racemic mixture, in this study we focused on armodafinil (the R-isomer), which is believed to be the active enantiomer, to eliminate any extraneous effects from esmodafinil (the S-isomer). While recent work has pointed to an important role for dopaminergic neurotransmission, including the specific possibility of dopaminedependent adrenergic signaling in modafinil's mechanism of action, ${ }^{5,8}$ the neuronal site(s) at which this occurs remains undetermined. As sleep-promoting VLPO neurons are inhibited by dopamine via activation of alpha- 2 adrenoceptors ${ }^{14}$ and noradrenergic inhibition of VLPO neurons is potentiated by modafinil in vitro, ${ }^{13}$ we hypothesized that modafinil might promote wake through one or both of these mechanisms at the level of the VLPO neurons in vivo. The results of the present study indicate that animals with large VLPO lesions demonstrate increased wakefulness at baseline, and when treated with either amphetamine or armodafinil have a smaller increase in wake compared to intact animals, but reach the same level of total wakefulness. Thus, our results are consistent with one of the effects of armodafinil and methamphetamine being to shut down the VLPO, but that both drugs must have additional effects that account for the increase in wakefulness beyond baseline in the VLPOx animals.

In early work investigating potential neuronal targets for modafinil, Engber et al ${ }^{18}$ showed an increase in c-Fos expression in several hypothalamic and limbic structures, including the central nucleus of the amygdala, following modafinil administration in rats. Lin et $\mathrm{al}^{19}$ also studied the pattern of c-Fos activation after modafinil administration, but in cats, and emphasized increased expression in the anterior hypothalamic area. A later study in rats, ${ }^{15}$ which utilized a more sensitive c-Fos antiserum, found a much more extensive pattern of c-Fos expression after modafinil administration, including many neurons in arousalpromoting cell groups (such as the tuberomammillary nucleus and orexin neurons in the lateral hypothalamus), but also in the striatum, including the caudate-putamen and nucleus accumbens. This group also reported a reduction in c-Fos in the VLPO. Because the VLPO acts to decrease wake and the putative action of modafinil in the VLPO would be inhibitory, a decrease in c-Fos labeling is consistent with modafinil-mediated suppression of VLPO neuronal activity.

In the present study we evaluated c-Fos expression across the neuraxis in response to armodafinil or methamphetamine administration with the working hypothesis that the different 
behavioral profiles we observed post-drug administration (ie, armodafinil versus methamphetamine) would be reflected in differential CNS c-Fos expression. This, in turn, would possibly reveal candidate CNS structure(s) beyond the VLPO that are critical for mediating armodafinil's wake-promoting properties. We in fact observed virtually no differences in c-Fos expression across CNS nuclei between armodafinil and methamphetamine treated animals. While the c-Fos finding is difficult to reconcile with the markedly different behavioral profiles observed post-injection, it may simply reflect a limitation of the c-Fos technique, which may not stain all activated cell groups. Alternatively, the degree to which armodafinil and methamphetamine activate c-Fos stained cell groups may not be reflected in the counts due to a ceiling effect (ie, maximal c-Fos expression at a less than maximum level of physiological activity).

Similar, however, to Scammell et al, ${ }^{15}$ our c-Fos results did differ from a previous report in cats ${ }^{19}$ insofar as we found near equivalent c-Fos expression in the striatum following armodafinil or methamphetamine injections at the same dosages used in the EEG-based work in the present study (Figure 3). The activation of neurons in the striatum is not typical of untreated waking rats, but is almost certainly due to activation of D1 receptors which cause an increase in intracellular cyclic adenosine monophosphate (cAMP), one of the key drivers of c-Fos expression. ${ }^{20}$ On the other hand, D2 receptor-bearing striatal neurons would not be expected to show c-Fos in response to dopaminergic stimulation, as the D2 receptor causes a decrease in intracellular cAMP. ${ }^{21}$ Nevertheless, despite showing no c-Fos, inhibition of the D2-receptive neurons would be consistent with increased wake as well, as activation of these neurons in the nucleus accumbens by the $\mathrm{A} 2 \mathrm{a}$ adenosine receptor is thought to cause sleepiness, and blockade of the A2a receptor by caffeine to cause wakefulness. ${ }^{22}$ Thus, the c-Fos seen in the striatum after modafinil may actually be in the neurons that are selectively not related to its wake-promoting effect. Given the current view of the role of striatal dopaminergic transmission in addictive behaviors, ${ }^{23}$ these findings raise the interesting question of why (ar)modafinil's abuse potential and addictive properties are so much lower than that of methamphetamine, ${ }^{24,25}$ which is an important area of future research.

Other studies have employed a wide range of methodologies including chemical toxins, neural lesions, and genetically modified mice in an attempt to determine the CNS site(s) via which modafinil exerts its wake-promoting influence. Similar to the present study, however, most of these studies have yielded surprisingly limited insight. For example, it was reported that chemical ablation of noradrenergic forebrain projections from the locus coeruleus (LC), an aminergic nucleus implicated in sleep-wake regulation, did not block the wake-promoting effects of modafinil in vivo nor, in fact, was baseline sleep altered. ${ }^{8}$ Similarly, bilateral, electrolytic lesions in rats of the central nucleus of the amygdala, a limbic region strongly activated by modafinil, also failed to attenuate the waking response to modafinil administration nor, again, was baseline sleep altered. ${ }^{26}$ Modafinil also effectively increased wakefulness in orexin null mice. ${ }^{27}$ Interestingly, mice lacking the melanin-concentrating hormone $(\mathrm{MCH})$ type I receptor are less sensitive to modafinil's wake-promoting properties ${ }^{28}$ whereas dopamine transporter (DAT) knockout mice are completely unresponsive to modafinil administration. ${ }^{5}$ It was more recently shown that both D1 and D2 receptors are necessary for mediating the arousal effect of modafinil. ${ }^{29}$ Given, however, the broad CNS distribution of MCH type I receptors, DAT, and D1/D2 receptors, ranging from cortical and limbic to striatal, hypothalamic and brainstem targets, our findings do not identify specific CNS targets of modafinil or amphetamine.

One clue may come from the recent studies showing that caffeine causes increased locomotor activity in mice by its action blocking A2a adenosine receptors in the nucleus accumbens (NAc). ${ }^{22}$ Caffeine is a psychostimulant with similar wake-promoting and fatigue-countering properties as modafinil. As the NAc also contains D1 and D2 receptors, this basal ganglia structure may be a key target of modafinil. Recent work has suggested that this may indeed be the case. ${ }^{30}$

\section{Conclusion}

The results of the present study show that, while inhibition of VLPO neurons is potentially one of the mechanisms by which armodafinil and methamphetamine exert their wakepromoting effects, these compounds also likely act directly on wake-promoting circuitry.

\section{Acknowledgments}

This study was supported by grants from Cephalon Inc, and by National Institute of Health Grants NS073613, NS074205 and AG09975, and the G Harold and Leila Y Mathers Foundation. We are grateful to Quan Hue Ha for excellent technical assistance.

\section{Disclosure}

The authors report no conflicts of interest in this work. 


\section{References}

1. Scammell TE, Matheson J. Modafinil: a novel stimulant for the treatment of narcolepsy. Expert Opin Investig Drugs. 1998;7(1):99-112.

2. Minzenberg MJ, Carter CS. Modafinil: a review of neurochemical actions and effects on cognition. Neuropsychopharmacology. 2008;33(7):1477-1502.

3. Harsh JR, Hayduk R, Rosenberg R, et al. The efficacy and safety of armodafinil as treatment for adults with excessive sleepiness associated with narcolepsy. Curr Med Res Opin. 2006;22(4):761-774.

4. Saper CB, Scammell TE. Modafinil: a drug in search of a mechanism. Sleep. 2004;27(1):11-12.

5. Wisor JP, Nishino S, Sora I, Uhl GH, Mignot E, Edgar DM. Dopaminergic role in stimulant-induced wakefulness. J Neurosci. 2001;21(5):1787-1794.

6. Lin JS, Roussel B, Akaoka H, Fort P, Debilly G, Jouvet M. Role of catecholamines in the modafinil and amphetamine induced wakefulness, a comparative pharmacological study in the cat. Brain Res. 1992;591(2):319-326.

7. Duteil J, Rambert FA, Pessonnier J, Hermant JF, Gombert R, Assous E. Central alpha 1-adrenergic stimulation in relation to the behaviour stimulating effect of modafinil; studies with experimental animals. Eur J Pharmacol. 1990;180(1):49-58.

8. Wisor JP, Eriksson KS. Dopaminergic-adrenergic interactions in the wake promoting mechanism of modafinil. Neuroscience. 2005;132(4): 1027-1034.

9. Sherin JE, Shiromani PJ, McCarley RW, Saper CB. Activation of ventrolateral preoptic neurons during sleep. Science. 1996;271(5246): 216-219.

10. Lu J, Greco MA, Shiromani P, Saper CB. Effect of lesions of the ventrolateral preoptic nucleus on NREM and REM sleep. J Neurosci. 2000;20(10):3830-3842.

11. Vetrivelan R, Fuller PM, Yokota S, Lu J, Saper CB. Metabolic effects of chronic sleep restriction in rats. Sleep. 2012;35(11):1511-1520.

12. Saper CB, Fuller PM, Pedersen NP, Lu J, Scammell TE. Sleep state switching. Neuron. 2010;68(6):1023-1042.

13. Gallopin T, Luppi PH, Rambert FA, Frydman A, Fort P. Effect of the wake-promoting agent modafinil on sleep-promoting neurons from the ventrolateral preoptic nucleus: an in vitro pharmacologic study. Sleep. 2004;27(1):19-25.

14. Cornil CA, Balthazart J, Motte P, Massotte L, Seutin V. Dopamine activates noradrenergic receptors in the preoptic area. $J$ Neurosci. 2002;22(21):9320-9330.

15. Scammell TE, Estabrooke IV, McCarthy MT, et al. Hypothalamic arousal regions are activated during modafinil-induced wakefulness. J Neurosci. 2000;20(22):8620-8628.

16. Paxinos $G$, Watson $C$. The rat brain in stereotaxic coordinates. Fifth edition: Elsevier, 2004.
17. Fuller PM, Sherman D, Pedersen NP, Saper CB, Lu J. Reassessment of the structural basis of the ascending arousal system. J Comp Neurol. 2011;519(5):933-956.

18. Engber TM, Koury EJ, Dennis SA, Miller MS, Contreras PC, Bhat RV. Differential patterns of regional c-Fos induction in the rat brain by amphetamine and the novel wakefulness-promoting agent modafinil. Neurosci Lett. 1998;241(2-3):95-98.

19. Lin JS, Hou Y, Jouvet M. Potential brain neuronal targets for amphetamine-, methylphenidate-, and modafinil-induced wakefulness, evidenced by c-fos immunocytochemistry in the cat. Proc Natl Acad Sci US A. 1996;93(24):14128-14133.

20. Foulkes NS, Laoide BM, Schlotter F, Sassone-Corsi P. Transcriptional antagonist cAMP-responsive element modulator (CREM) downregulates c-fos cAMP-induced expression. Proc Natl Acad Sci U S A. 1991;88(12):5448-5452.

21. Neves SR, Ram PT, Iyengar R. G protein pathways. Science. 2002; 296(5573):1636-1639.

22. Lazarus M, Shen HY, Cherasse Y, et al. Arousal effect of caffeine depends on adenosine $\mathrm{A} 2 \mathrm{~A}$ receptors in the shell of the nucleus accumbens. J Neurosci. 2011;31(27):10067-10075.

23. Everitt BJ, Robbins TW. Neural systems of reinforcement for drug addiction: from actions to habits to compulsion. Nat Neurosci. 2005; 8(11):1481-1489.

24. Deroche-Gamonet V, Darnaudéry M, Bruins-Slot L, Piat F, Le Moal M, Piazza PV. Study of the addictive potential of modafinil in naive and cocaine-experienced rats. Psychopharmacology (Berl). 2002;161(4): 387-395.

25. Myrick H, Malcolm R, Taylor B, LaRowe S. Modafinil: preclinical, clinical, and post-marketing surveillance - a review of abuse liability issues. Ann Clin Psychiatry. 2004;16(2):101-109.

26. Silvestri AJ, Sanford LD, Ross RJ, Mann GL, Pavlock A, Morrison AR. The central nucleus of the amygdala and the wake-promoting effects of modafinil. Brain Res. 2002;941(1-2):43-52.

27. Willie JT, Renthal W, Chemelli RM, et al. Modafinil more effectively induces wakefulness in orexin-null mice than in wild-type littermates. Neuroscience. 2005;130(4):983-995.

28. Adamantidis A, Salvert D, Goutagny R, et al. Sleep architecture of the melanin-concentrating hormone receptor 1-knockout mice. Eur $J$ Neurosci. 2008;27(7):1793-1800.

29. Qu WM, Huang ZL, Xu XH, Matsumoto N, Urade Y. Dopaminergic D1 and D2 receptors are essential for the arousal effect of modafinil. $J$ Neurosci. 2008;28(34):8462-8469.

30. Qiu MH, Liu W, Qu WM, Urade Y, Lu J, Huang ZL. The role of nucleus accumbens core/shell in sleep-wake regulation and their involvement in modafinil-induced arousal. PLoS One. 2012;7(9):e45471.
Nature and Science of Sleep

\section{Publish your work in this journal}

Nature and Science of Sleep is an international, peer-reviewed, open access journal covering all aspects of sleep science and sleep medicine, including the neurophysiology and functions of sleep, the genetics of sleep, sleep and society, biological rhythms, dreaming, sleep disorders and therapy, and strategies to optimize healthy sleep. The journal welcomes
Dovepress

original research, clinical \& epidemiological studies, reviews \& evaluations, case reports and extended reports. The manuscript management system is completely online and includes a very quick and fair peerreview system, which is all easy to use. Visit http://www.dovepress.com/ testimonials.php to read real quotes from published authors. 